nephron

Practice
Nephron 2016;132:312-316

DOI: $10.1159 / 000444456$
Received: October 27, 2015

Accepted after revision: February 1, 2016

Published online: March 3, 2016

\title{
Timely Diagnosis of Acute Kidney Injury Using Kinetic eGFR and the Creatinine Excretion to Production Ratio, E/eG - Creatinine Can Be Useful!
}

\author{
Zoltán H. Endre ${ }^{a-c}$ Timothy J. Pianta ${ }^{a, b}$ John W. Pickering ${ }^{c, d}$ \\ a Department of Nephrology, Prince of Wales Hospital, and bPrince of Wales Clinical School, University of New \\ South Wales, Sydney, Australia; ' Department of Medicine, University of Otago, and d Department of Emergency, \\ Christchurch Hospital, Christchurch, New Zealand
}

\section{Key Words}

GFR under non-steady state conditions - Delayed graft function · Kinetic eGFR · Creatinine excretion - Creatinine production

\section{Abstract \\ Post transplant repeated measurements of urine volume and serum creatinine $(\mathrm{s} C \mathrm{C}$ ) are used to assess kidney func- tion. Under non-steady state conditions, repeated measure- ment of $\mathrm{s} C r$ allows calculation of the kinetic estimated GFR (KeGFR). Additional measurement of urinary creatinine al- lows the calculation of the creatinine excretion to (estimat- ed) production ratio $(\mathrm{E} / \mathrm{eG})$. We hypothesized that post- transplant KeGFR and E/eG would predict delayed graft function (DGF), as early as $4 \mathrm{~h}$ and outperform a validated clinical model at $12 \mathrm{~h}$. This was a retrospective analysis of prospectively acquired data in a study of 56 recipients of de- ceased-donor kidney transplant. We assessed predictive performance with the area under the receiver operator char- acteristic curve (AUC) and the added value to a clinical mod- el with integrated discrimination improvement analysis. At $4 \mathrm{~h}$, the AUC for E/eG was $0.87(95 \% \mathrm{Cl} 0.77-0.96)$ and for}

KeGFR 0.69 (95\% Cl 0.56-0.83). Both E/eG and KeGFR improved the risk prediction of a clinical model for DGF by 32 and $18 \%$, and for non-DGF by 17 and $10 \%$, respectively. While E/eG had better predictive performance of DGF than KeGFR, KeGFR might also facilitate perioperative management including drug dosing after kidney transplantation. Together these measurements may facilitate the possibility of conducting trials of early intervention to ameliorate the adverse effects of ischaemia-reperfusion injury on long-term DGF.

(c) 2016 S. Karger AG, Basel

\section{Introduction}

Approximately $20-30 \%$ of deceased donor kidneys and half the kidneys donated after cardiac death develop delayed graft function (DGF) [1]. DGF is usually defined

Contribution from the AKI and CRRT 2015 Symposium at the 20th International Conference on Advances in Critical Care Nephrology, Manchester Grand Hyatt, San Diego, Calif., USA, February 17-20, 2015.

\section{KARGER}

(c) 2016 S. Karger AG, Basel

$1660-8151 / 16 / 1324-0312 \$ 39.50 / 0$

E-Mail karger@karger.com

www.karger.com/nef
Prof. Zoltán H. Endre

Department of Nephrology

Prince of Wales Hospital

High Street, Randwick, Sydney NSW 2031 (Australia)

E-Mail z.endre@unsw.edu.au 
as a requirement for dialysis within 1 week after transplantation. In addition to requiring dialysis, DGF is associated with increased rates of rejection and graft loss, inferior GFR and longer hospital stays. Early identification of patients with DGF is particularly important for timely modification of immunosuppression (especially calcineurin inhibitors) and potentially toxic drugs including valgan- (or vala-) ciclovir and or co-trimoxazole used for the prevention of Cytomegalovirus and Pneumocystis jirovecii infection respectively after transplantation.

There are substantial problems associated with the use of serum creatinine ( $\mathrm{sCr}$ ) in the diagnosis of acute kidney injury (AKI) and in the recognition of renal functional recovery. These include a long half-life, which delays accurate estimation of GFR after change and delays diagnosis $[2,3]$. The usefulness of creatinine would be enhanced if one could shorten the time frame to detect change in renal function under non-steady state conditions. Intuitively, examining the velocity of change of $\mathrm{sCr}$ in the early hours following transplant might predict the subsequent need for dialysis. This is the basis of kinetic eGFR (KeGFR) [4]. It has been established that KeGFR predicts DGF and improved on existing risk prediction models (e.g. [5]). The KeGFR formula is potentially adaptable to other filtration biomarkers including plasma cystatin C [1]. Also intuitive is the suggestion that if the rate of excretion (E) is less than the rate of production (estimated generation, eG) of creatinine, then the ratio $(\mathrm{E} / \mathrm{eG})$ would be a near 'real-time' marker of kidney performance [6] relative to steady state performance, and therefore a predictor of DGF.

This analysis compares KeGFR with E/eG and illustrates their use under non-steady state conditions in a situation where some degree of AKI is inevitable, namely, kidney transplantation. We present here the main findings from a recent retrospective application of the KeGFR formula in deceased-donor kidney transplant recipients [1]. This includes an analysis of whether KeGFR adds value to a validated clinical risk prediction model [5]. In a new analysis, we compare the prognostic performance of KeGFR with that of E/eG.

\section{Methods}

This was a retrospective analysis of prospectively acquired data from deceased-donor kidney transplant recipients from a single centre [1]. All patients received a uniform protocol of immunosuppression with corticosteroids, basiliximab, and mycophenolate sodium, and tacrolimus or cyclosporine at the treating physician's discretion. Urine volume was measured hourly. sCr and uCr were measured immediately post transplant and at 4,8 , and $12 \mathrm{~h}$.
The KeGFR was calculated from the change in consecutive values of $\mathrm{sCr}$, and the estimated creatinine production rate and volume of distribution (Vd) to estimate GFR was calculated according to the formula derived by Chen [4]:

$$
\text { KeGFR }=\frac{\text { BsCr } \times \text { eGFR }}{\text { mean sCr }} \times\left(1-\frac{24 \times \Delta \mathrm{sCr}}{\Delta \mathrm{t}(\mathrm{h}) \times \frac{\max \Delta \mathrm{sCr}}{\text { day }}}\right)
$$

Accounting for: baseline sCr $(\mathrm{BsCr})$; the corresponding unadjusted eGFR (eGFR, determined by the CKD-EPI formula); the mean of 2 consecutive values of sCr (mean sCr e.g. at 4 and $8 \mathrm{~h}$ ); the difference between each 2 values $(\Delta s \mathrm{Cr})$; the time interval between samples ( $\Delta \mathrm{t}$ hours), and the estimated increase in $\mathrm{sCr}$ in 1 day when GFR is zero $(\max \Delta \mathrm{sCr} /$ day $)$. Further details of the derivation of the formula can be found in Chen [4] and Pianta et al. [1]. For KeGFR, the first term represents the daily creatinine production divided by the mean $\mathrm{sCr}$ concentration, which is therefore equal to GFR when production equals excretion; the second term is a correction factor accounting for the change in sCr between 2 samples, the time over which change occurs, and the maximum increase in biomarker concentration in the allocated time, which can be measured or a historical approximation can be used, for example, $1.5 \mathrm{mg} / \mathrm{dl}(133 \mu \mathrm{mol} / \mathrm{l})$ in $24 \mathrm{~h} \mathrm{[4]}$. When sCr increases at its predicted maximal rate over any period of time, the subtracted term equals 1 , and the KeGFR is zero. When sCr is in a steady state, $\Delta \mathrm{B}_{\mathrm{c}}$ is zero, the subtracted term equals zero, and KeGFR simply equals eGFR. When sCr decreases, $\Delta \mathrm{B}_{\mathrm{c}}$ and thus the subtracted term is a negative number. The subtraction of a negative value from 1 produces a factor $>1$ and the KeGFR exceeds the arithmetic mean of eGFR produced by the first half of the equation. A very rapid fall in sCr produces a very high value of KeGFR. One way to view the KeGFR is as an estimate of GFR at the arithmetic midpoint between $2 \mathrm{sCr}$ values [1].

The creatinine excretion to production ratio, E/eG, was calculated at 4,8 , and $12 \mathrm{~h}$, using the previous $4 \mathrm{~h}$ of creatinine excretion. Excretion (E) is simply urinary creatinine concentration multiplied by urinary volume divided by collection time. eG was estimated according to the formula of Bjornsson [7] as previously [6]: $\mathrm{eG}=\left(\mathrm{A}-\mathrm{B}^{*} \text { age }(\text { years })\right)^{*}$ weight $(\mathrm{kg}) / 24$, where for males $\mathrm{A}=27$ and $B=0.173$, and for females $A=25, B=0.175$. In steady state conditions, excretion equals production and the ratio is 1 . A value $<1$ indicates sub-optimal kidney function. We hypothesised that patients with DGF would have E/eG much $<1$ and lower than that of patients without DGF.

The reference clinical model was derived from 20 parameters related to the recipient ( 9 parameters, e.g. age, sex), donor (8 parameters, e.g. age, terminal $\mathrm{sCr}$ ), and transplantation ( 3 parameters e.g. cold ischaemia time) as previously described [5] and subsequently validated [8]. A logistic regression analysis calculated the probability of DGF for each patient.

DGF was defined as the requirement for dialysis within 1 week after transplantation.

\section{Statistical Analysis}

The predictive performance of KeGFR, E/eG and the clinical model was assessed by the receiver operator characteristic analysis to determine the area under the curve (AUC). The added value of 
Table 1. Prediction of DGF in recipients of kidneys from deceased kidney donors

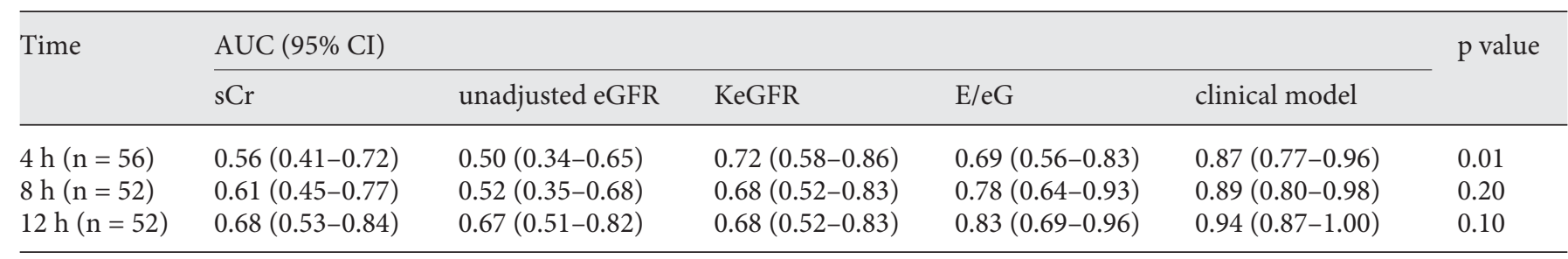

AUC and 95\% CIs for prediction of delayed sCr, unadjusted eGFR, KeGFR, the creatinine excretion to production ratio (E/eG), and the clinical model based on donor and recipient factors. p values compare the AUCs of E/eG with KeGFR.

the KeGFR and E/eG to the validated clinical model was assessed by the integrated discrimination improvement (IDI) [9] and risk assessment plots [10]. The IDI is presented separately for those with DGF (IDI-DGF) and represents the increase in mean risk over the clinical model; and for patients without DGF (IDI-nonDGF) it represents the mean decrease in risk from the clinical model in patients without DGF. Analysis was with the Matlab version R2013b (Mathworks, Natick, Mass., USA) and R version 3.2.2 (http://www.R-project.org/).

\section{Results}

Fifty-six patients were recruited, of whom 22 (39\%) patients developed DGF (for patient characteristics, see [1]).

At $4 \mathrm{~h}$ post transplant, KeGFR predicted DGF with an AUC of 0.69 (95\% CI 0.56-0.83) similar to the AUC for the clinical model, 0.72 (95\% CI 0.58-0.86; table 1).

At $4 \mathrm{~h}, \mathrm{E} / \mathrm{eG}$ was $<1$ for all patients indicating that creatinine excretion had yet to equilibrate with production. However, E/eG was lower in patients with DGF than nonDGF and the AUC was good, 0.87 (95\% CI 0.77-0.96), and greater than that of KeGFR $(\mathrm{p}=0.01)$.

The AUCs for both KeGFR and E/eG increased at latter time points, and were greater for $\mathrm{E} / \mathrm{eG}$ at each time point (table 1).

The addition of KeGFR to the clinical model improved risk prediction of those both with DGF (IDIDGF $=0.18(95 \%$ CI 0.04-0.35)) and those without DGF $($ IDI-non-DGF $=0.10(95 \% \mathrm{CI} 0.03-0.21))$ at $12 \mathrm{~h}$. A risk assessment plot (fig. 1b), illustrates that KeGFR increased risk for those with DGF for whom the calculated risk of the clinical model was $>0.3$, and decreased risk for those without DGF for whom the calculated risk was $<0.5$.

The addition of E/eG to the clinical model better improved risk prediction of those both with DGF (IDI-
$\mathrm{DGF}=0.32(95 \%$ CI $0.17-0.51))$ and those without DGF $($ IDI-non-DGF $=0.17(95 \%$ CI $0.07-0.30))$ at $12 \mathrm{~h}$ (fig. 1c).

\section{Discussion}

An estimation of GFR under non-steady state conditions by the KeGFR formula predicted DGF within $4 \mathrm{~h}$ of kidney transplantation. The AUC was modest suggesting limited utility. However, the AUC was greater at later time points, which could still be considered early post transplant. Furthermore, KeGFR independently enhanced the clinical model for DGF prediction at 4,8 , and $12 \mathrm{~h}$ after transplantation. By contrast, neither sCr concentrations nor unadjusted eGFR improved the prediction of DGF. This was as expected since these measures account for neither preoperative variability due to dialysis, nor the non-steady state after transplantation.

The creatinine excretion to estimated production ratio E/eG was a better predictor of DGF within $4 \mathrm{~h}$ of transplantation with a good AUC, which suggests likely clinical utility. As for KeGFR, the AUC improved at later time points and the ratio added value to a clinical model by increasing the mean calculated risk of those with DGF by $32 \%$ and decreasing the mean calculated risk of those who did not have DGF by $17 \%$.

While both KeGFR and E/eG reflect the state of the kidney over a few hours of urine collection $(\mathrm{E} / \mathrm{eG})$ and between plasma creatinine measurements (KeGFR), only KeGFR is likely to be useful to help adjust medication dosages. E/eG estimates the extent to which excretion matches production, and KeGFR estimates GFR.

This is the first analysis of KeGFR or E/eG in transplant patients. KeGFR has compared favorably to novel kidney damage biomarkers for prediction of short-term 


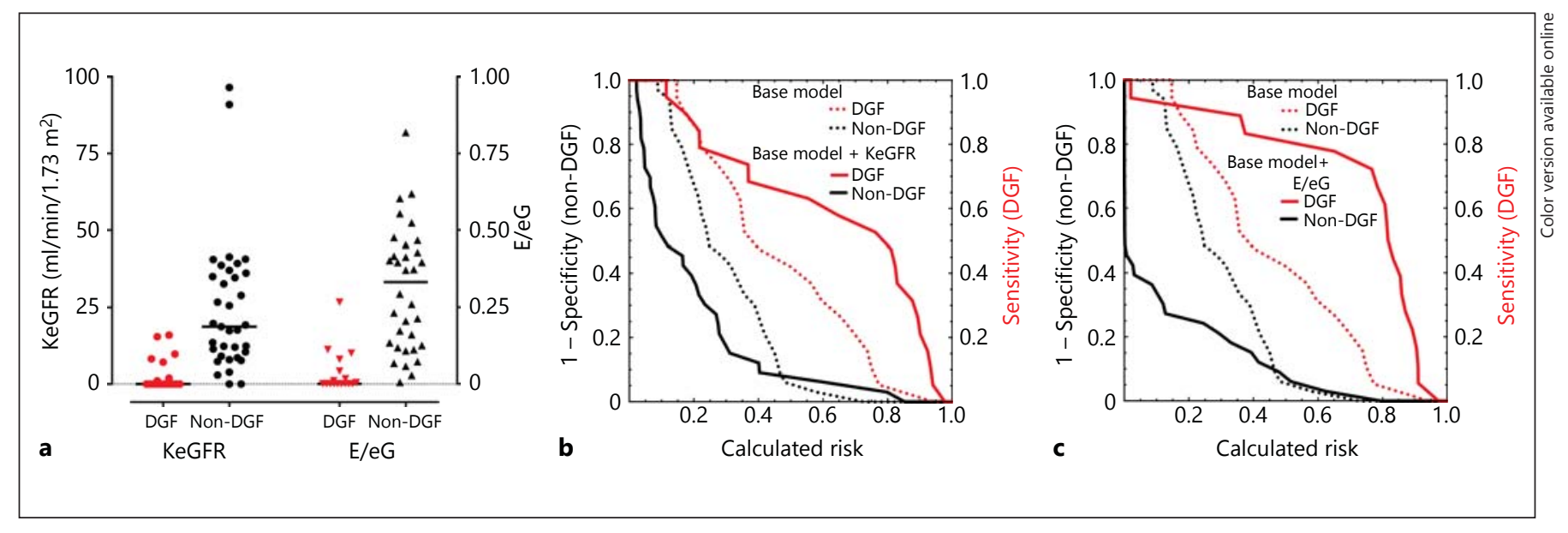

Fig. 1. The added value of KeGFR and E/eG at $12 \mathrm{~h}$ post-transplant. a KeGFR and E/eG at $12 \mathrm{~h}$ for patients with DGF (red) and nonDGF (black). b Clinical model enhancement by KeGFR at $12 \mathrm{~h}$ (adapted from [1]). The risk assessment plot shows the base clinical model (dotted lines) and the model after addition of KeGFR (solid lines). Red lines are sensitivity vs. the calculated risk for patients who developed DGF. Black lines represent 1 - specificity vs. the calculated risk for those who did not have DGF. Improved risk assessment is demonstrated by movement of the red curve to the top-right corner and black curve to the bottom left corner after addition of the KeGFR to the clinical model, that is, separation of curves. The value of the IDI is shown by the area between the respective solid and dotted lines (for discussion of risk assessment plots, see [10]. c Clinical model enhancement by E/eG at $12 \mathrm{~h}$. The risk assessment plot shows the base clinical model (dotted lines) and the model after addition of E/eG (solid lines). recovery from AKI and major adverse kidney events intensive care patients [11]. E/eG predicted AKI and identified recovered AKI patients in ICU patients [6].

While applying E/eG may also facilitate the early prediction of DGF, unlike KeGFR, its expression is in unfamiliar units, and is therefore not intuitive to clinicians already familiar with eGFR. Further research may establish a clinically applicable threshold. For example, in this dataset at $4 \mathrm{~h}$, a threshold E/eG of 0.15 had $95 \%$ sensitivity and $70 \%$ specificity. Furthermore, KeGFR may facilitate improved postoperative drug dosing. Therefore, although E/eG outperformed KeGFR, we recommend measuring both.

Whether applied after transplantation, or to evaluate $\mathrm{AKI}$ and its recovery, evaluation of KeGFR or E/eG each requires repeated measurement of functional biomarkers, with careful documentation of the time of sample collection.

The data presented here are based on retrospective analysis and a small single-centre study and requires validation in a much larger cohort. Further methodological limitations in each method include the assumptions of single-compartment kinetics, and no change in creatinine production or Vd due to plasma volume expansion [1]. Neither technique addresses whether improved GFR or creatinine excretion in those without DGF represents the recovery of baseline donor function or recruitment of renal reserve.

This study illustrates the principle that sCr measurements, which can be obtained at modest cost, remain useful to guide clinical trials of early management in some types of AKI if performed frequently enough and used in a manner that accounts for the non-steady state. The predictive value of clinical risk prediction models can be improved by adding a KeGFR derived from repeated creatinine measurement or the E/eG ratio derived from the urine output and urine creatinine measurement within $4 \mathrm{~h}$ of transplantation.

References
1 Pianta TJ, Endre ZH, Pickering JW, Buckley NA, Peake PW: Kinetic estimation of GFR improves prediction of dialysis and recovery after kidney transplantation. PLoS One 2015; 10:e0125669.

2 Endre ZH, Pickering JW, Walker RJ: Clearance and beyond: the complementary roles of GFR measurement and injury biomarkers in acute kidney injury (AKI). Am J Physiol Renal Physiol 2011;301:F697-F707.

-3 Chew-Harris JS, Florkowski CM, Elmslie JL, Livesey J, Endre ZH, George PM: Lean mass modulates glomerular filtration rate in males of normal and extreme body composition. Intern Med J 2014;44:749-756. 
4 Chen S: Retooling the creatinine clearance equation to estimate kinetic GFR when the plasma creatinine is changing acutely. J Am Soc Nephrol 2013;24:877-888.

$\checkmark 5$ Irish WD, Ilsley JN, Schnitzler MA, Feng S, Brennan DC: A risk prediction model for delayed graft function in the current era of deceased donor renal transplantation. Am J Transplant 2010;10:2279-2286.

-6 Pickering JW, Mellas J: A simple method to detect recovery of glomerular filtration rate following acute kidney injury. Biomed Res Int 2014;2014:542069.
Bjornsson TD: Use of serum creatinine concentrations to determine renal function. Clin Pharmacokinet 1979;4:200-222.

-8 Rodrigo E, Miñambres E, Ruiz JC, Ballesteros A, Piñera C, Quintanar J, et al: Prediction of delayed graft function by means of a novel web-based calculator: a single-center experience. Am J Transplant 2012;12:240 244.
9 Pencina MJ, D’Agostino RB Sr, D’Agostino RB Jr, Vasan RS: Evaluating the added predictive ability of a new marker: from area under the ROC curve to reclassification and beyond. Stat Med 2008;27:157-172.

10 Pickering JW, Endre ZH: New metrics for assessing diagnostic potential of candidate biomarkers. Clin J Am Soc Nephrol 2012;7: 1355-1364.

11 Dewitte A, Joannès-Boyau O, Sidobre C, Fleureau C, Bats ML, Derache P, et al: Kinetic eGFR and novel AKI biomarkers to predict renal recovery. Clin J Am Soc Nephrol 2015; 10:1900-1910. 


\section{Erratum}

In the article by Endre et al., entitled 'Timely diagnosis of acute kidney injury using kinetic eGFR and the creatinine excretion to production ratio, E/eG - Creatinine can be useful!' [Nephron 2016;132:312-316, DOI: 10.1159/000444456], table 1 has a serious mistake. The AUCs are not in agreement with those mentioned in the Results section. The values of E/eG appeared as those of the clinical model and the values of KeGFR appeared as those of E/eG. The correct table should be as follows:

Table 1. Prediction of DGF in recipients of kidneys from deceased kidney donors

\begin{tabular}{lllllll}
\hline Time & AUC $(95 \% \mathrm{CI})$ & & & & \\
\cline { 2 - 5 } & $\mathrm{sCr}$ & eGFR & clinical model & KeGFR & E/eG & \\
\hline $4 \mathrm{~h}(\mathrm{n}=56)$ & $0.56(0.41-0.72)$ & $0.50(0.34-0.65)$ & $0.72(0.58-0.86)$ & $0.69(0.56-0.83)$ & $0.87(0.77-0.96)$ & 0.01 \\
$8 \mathrm{~h}(\mathrm{n}=52)$ & $0.61(0.45-0.77)$ & $0.52(0.35-0.68)$ & $0.68(0.52-0.83)$ & $0.78(0.64-0.93)$ & $0.89(0.80-0.98)$ & 0.20 \\
$12 \mathrm{~h}(\mathrm{n}=52)$ & $0.68(0.53-0.84)$ & $0.67(0.51-0.82)$ & $0.68(0.52-0.83)$ & $0.83(0.69-0.96)$ & $0.94(0.87-1.00)$ & 0.10 \\
\hline
\end{tabular}

AUCs (with 95\% CI) for prediction of delayed graft function using sCr, unadjusted eGFR, the clinical model based on donor and recipient factors, KeGFR, and the creatinine excretion to production ratio (E/eG). p values compare the AUCs of E/eG with KeGFR. 